\title{
Designing an Optimal Lightning Protection Scheme for Substations Using Shielding Wires
}

\author{
Abolfazl Khodadadi \\ Electrical Engineering Department \\ Amirkabir University of Technology \\ Tehran, Iran \\ a.khodadadi@aut.ac.ir
}

\author{
Mohammad Hassan Nazari \\ Electrical Engineering Department \\ Amirkabir University of Technology \\ Tehran, Iran \\ nazary@aut.ac.ir
}

\author{
Seyed Hossein Hosseinian \\ Electrical Engineering Department \\ Amirkabir University of Technology \\ Tehran, Iran \\ hosseinian@aut.ac.ir
}

\begin{abstract}
An optimal lightning protection scheme for a substation using shielding wires is investigated in this paper through computer software analysis. An economic approach is utilized by choosing a reasonable trade-off between protection, the number of shielding wires and the heights of them from the ground. This study is initially applied to a simple two-wire system and then extended to a sample substation. The solution for each problem is executed in MATLAB and 3-D realization is shown.
\end{abstract}

Keywords—substation protection; lightning stikes; shielding wires; computer aid analysis

\section{INTRODUCTION}

Faults and damages in substations are the culmination of sundry factors each of which play a significant role in the shielding failures among which lightning is one of the most important one. This phenomenon as a probabilistic event may cause severe consequences like power supply interruptions and economic losses [1-5]. Therefore, a lightning protection system is required for reducing the probability of a fault against direct lightning stroke to the objects and buses within the substations $[6,7]$. For more illustration on the lightening progress phenomenon, a brief description is presented in [8]. In the lightening, the stepped leader moves toward earth in stopping steps of about 50 meters. After each step, the stepped leader pauses, then proceeds along one or more paths. The time for each step is about $50 \mu$ s near the base of the cloud but decreases to about $13 \mu \mathrm{s}$ as it approaches the earth. The velocity of the stepped leader is slow, about $0.1 \%$ of the speed of light. As this stepped or downward leader reaches near the earth, an upward leader or return stroke start progressing to meet the downward leader. The speed of this stoke is about 10 to $30 \%$ of light which makes it possible to be observed by naked eye. The amplitude of this upward channel is more than $200 \mathrm{kA}$ with a median value of $33 \mathrm{kA}$. The total length of the downward leader or the upward channel is about 5 to $6 \mathrm{~km}$. The above description illustrates the mechanism of the first stoke of a lightning flash. One flash may consists of up to 54 stokes; however, the average is three stokes per flash

In order to apply protection to the equipment, three different methods can be utilized: shielding wires, masts and combination of these two. Each of the aforementioned methods for shielding a substation has its own advantages and disadvantages. However, the application of shielding wires is much more recommended due to its wide range of protection area [9]. In addition, the approach which is applied for lightning protection can be obtained from various equations, such as Young's equations, Love's equations, BrownWhitehead, IEEE-1992 and IEEE-1995 [8], [10]. For Young's equations only a small modification for the value of the strike distances is required and for Love's equations and IEEE-1995, there is no need for any adjustment. However, utilizing BrownWhitehead and IEEE-1992 needs significant changes in the value of different strike distances. In this paper, for the case of generality, Young's equations are employed.

MATLAB is employed in this paper for simulation. The economic aspect of the design is also considered and a trade-off between cost and level of security is proposed. Thus, this paper applies specific criteria for achieving the design with minimum possible cost.

\section{METHODOLOGY}

Different distances are used for defining the strike distance: (1) The strike distance to the shielding wire or shielding mast, $r_{s}(2)$ the strike distance to the object to be protected, $r_{c}$, and (3) the striking distance to the ground, $r_{g}$ [11]. All of these distances are shown in Figure 1. This figure also specifies the areas on which lightning strokes will be terminated. The following equations have been derived from the electromagnetic model to calculate the strike distance equations [8]:

$$
r_{s}=\gamma_{s} r_{g} \quad r_{c}=\gamma_{c} r_{g}
$$

in which $\gamma_{s}$ and $\gamma_{c}$ are coefficients which will be defined by Young's equations:

$$
\begin{array}{lll}
r_{g}=27 I^{0.32} & \text { for } h \geq 18 m & \text { otherwise } \gamma_{s}=1 \\
\gamma_{s}=\frac{444}{462-h} & \text { for } y \geq 18 m & \text { otherwise } \gamma_{c}=1
\end{array}
$$


Where $I$ is the design current in $\mathrm{kA}$ and $r_{s}, r_{g}$ and $r_{c}$ are strike distances in meters.

Design current is an important factor that highly affects the output parameters of the design. For practical designs, the design current value for voltages below $230 \mathrm{kV}$ is usually considered to be $5 \mathrm{kA}$ and for voltages above $230 \mathrm{kV}$ is considered to be $10 \mathrm{kA}$ [8], which is the value used in this paper.

In substations, the height of devices can vary from zero to the height of the shielding wires. Thus, the location of the objects and wires should be determined in such a way that a minimum number of shielding wires cover the entire area of the substation. Each shielding wire has a specific protection zone. For instance, consider a single wire as depicted in Figure 1. The actual area of the final protective zone depends on the number of shielding wires that have been employed. In order to clarify this issue, two cases are investigated as follows.

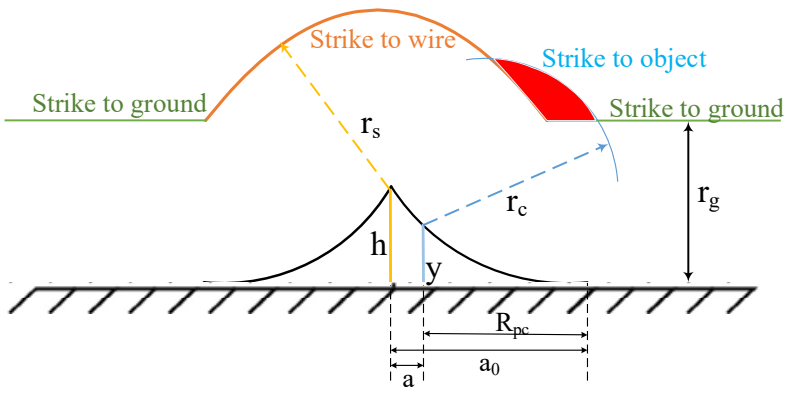

Fig. 1. Strike distances ( front view )

\section{A. One Shielding Wire}

In this case one shielding wire will be used in order to protect a specified zone. Assume that there is an object with height $y$ and a shielding wire at height $h$. Now if the position of the object from the shielding wire is known, with the following equations, the height of the shielding wire may be determined (see Fig. 1).

$$
\begin{aligned}
& R_{p o}=\sqrt{r_{c}^{2}-\left(r_{g}-y\right)^{2}} \\
& a_{0}=a+R_{p o} \\
& h=r_{g}-\sqrt{r_{s}^{2}-a_{0}^{2}}
\end{aligned}
$$

Where in these equations $a_{0}$ is the distance from the shielding wire to the object (Figure 2).

\section{B. Two Sheilding Wires}

The shielding zone which is introduced by two shielding wires is shown in Figure 3. The highlighted zone in this figure shows the protected area by two shielding wires. Having an object with the height of $y$, the horizontal distance of the protection, $a$, will be calculated as it follows:

$$
a=\sqrt{r_{c}^{2}-\left(r_{g}-h\right)^{2}}-\sqrt{r_{c}^{2}-\left(r_{g}-y\right)^{2}}
$$

Also the equations for calculating the horizontal protective distance between two shielding wires can be evaluated by (5) and is depicted in Figure 4.

$$
\begin{aligned}
& R_{p c}=\sqrt{r_{c}^{2}-\left(h-y+\sqrt{r_{s}^{2}-R_{c}^{2}}\right)^{2}} \\
& a_{c}=R_{c}-R_{p c}
\end{aligned}
$$

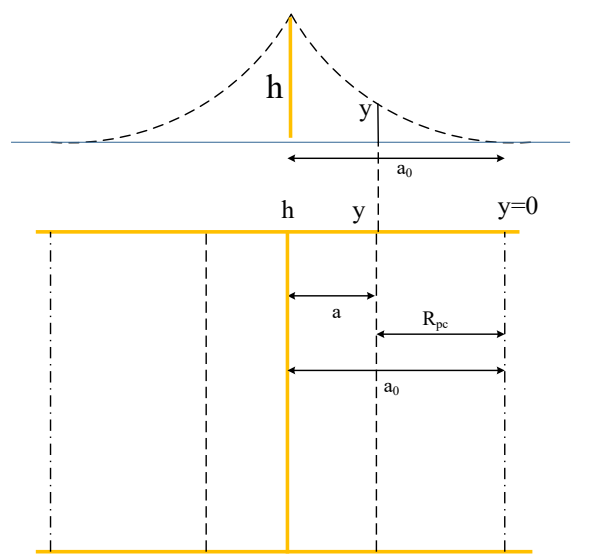

Fig. 2. Protective zone of a single wire ( upper view )

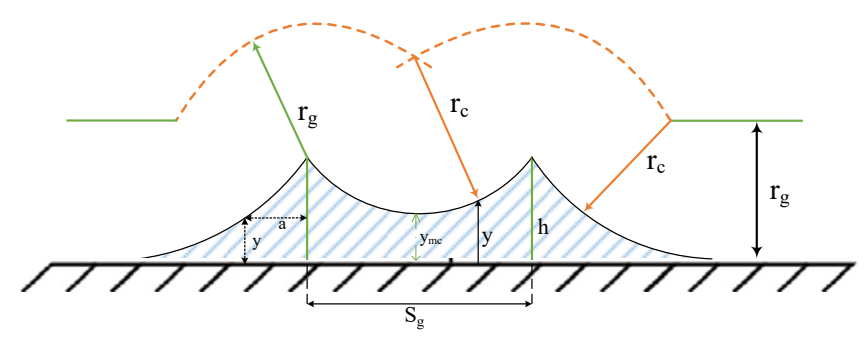

Fig. 3. Protected zone of the two shielding wires

The minimum height of the protection level is in the middle of the distance between two wires as shown in Figure 3 and it can be derived by setting the $R_{p c}$ equal to zero in (5), that is:

$$
y_{m c}=h-r_{c}+\sqrt{r_{s}^{2}-R_{c}^{2}}
$$

However, it should be mentioned that if the distance between two wires increases, the value of the $y_{m c}$ equals to zero and the configuration which is depicted in Figures 3 and 4 will not hold true, but the equations can be utilized.

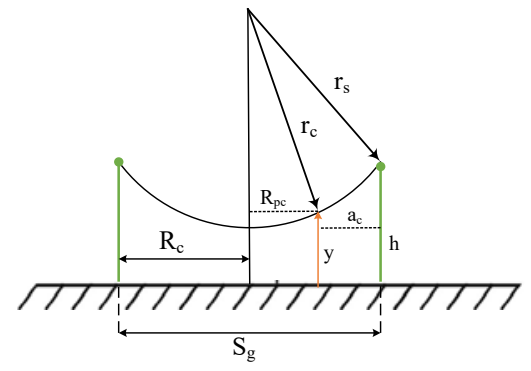

Fig. 4. Protective zone between two shield wires 
To clarify and visualize the shielding protection area by two wires, 3-D realization of Figure 4 is depicted in Figure 5.

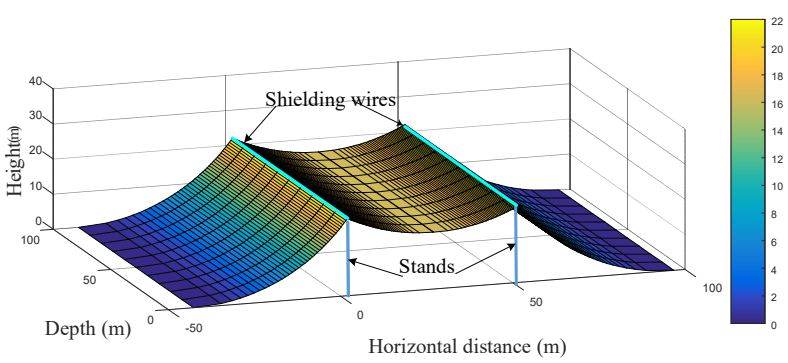

Fig. 5. 3-D visualization of shielding protection zone

\section{Economic Analysis}

The overall cost of the design should be calculated in a cost effective manner. So, a software should be employed to solve the problem with specific technical and economic constraints (number of wires and height are considered as parameters). As the height of the wires increases, the number of them decreases. An equation that can be applied for economic constraint can be defined as follows.

$$
\text { CostFunction }=\alpha \cdot \mathrm{N}+\beta . H
$$

Where $\alpha$ is a constant which transfers the number of wires, $N$, into monetary unit (\$/number) and $\beta$ is another constant which transfers the height of the wires (in meter) into monetary unit (\$/height). A person who is expert in this field should define these constants in order to have reasonable and economic solution.

\section{SimULATION RESUlTS}

\section{A. Case Study Information}

Any sample substation can be analyzed by the algorithm utilized for this paper. For instance, consider a substation which its front view is shown in Figure 6. The first part isa 345 $\mathrm{kV}$ transformer which its height is 30 feet (approximately 10 meter) or second part a $500 \mathrm{kV}$ EHV gas circuit breaker [12].

\section{B. Simulation Algorithm}

At the first stage, the algorithm puts two wires in the beginning and the end of the specified area at minimum acceptable height. Then it proceeds to calculate the shielding zone between these two wires. It is obvious that these placement cannot guarantee the full protection of the equipment between them. Then, it brings one of the wires closer to the other one in order to increase the height of the protection level between them. This process continues until the position of the second wire reaches a position ensuring that all of the equipment between them will be completely protected. The user can define the specific margin between the highest part of the equipment and the protection level defined by shielding wires. In this problem, the aforementioned margin is set one meter above the highest equipment.
After determining the position of the second wire, this process continues until the protection area of the last wire reaches the end of the defined area. The process which has been mentioned however, above will solve problem for a specific height. Next step is to increase the height of the wires and then repeat the same process. As the height of the wires increases, the number of them will decrease. Therefore, the economic criteria will be considered for the final scheme selection.

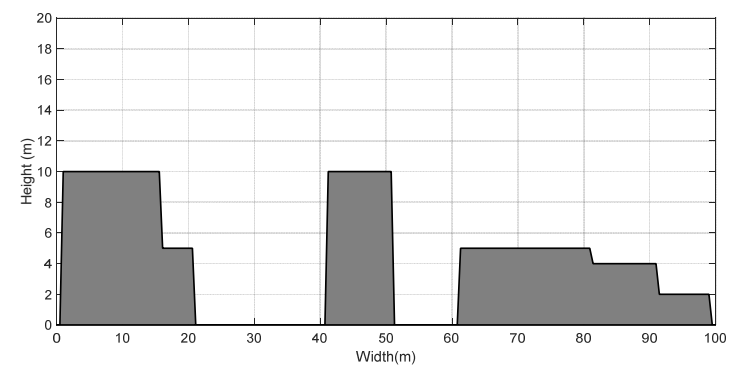

Fig. 6. Front view of the sample substation

\section{Simulation Results}

In the first part of the simulation, the minimum height of the wires is selected and the algorithm calculates the complete protection as depicted in Figure 7. As it was stated before, this design has been performed in a way that all equipment has at least one meter margin from the shielding area provided by the wires. In this case five shielding wires have been used and the height of the wires is 11.5 meter from the ground. The 3-D realization is shown in Figure 8.

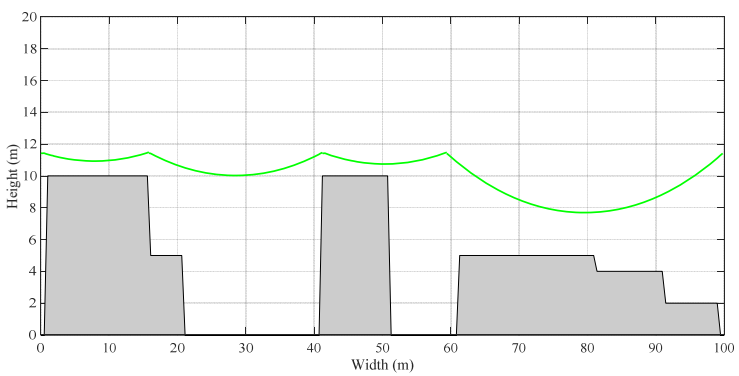

Fig. 7. Protection level provided by wires

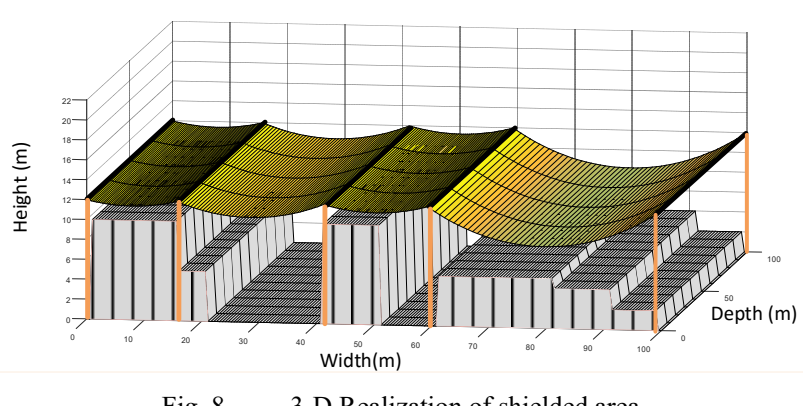

Fig. 8. 3-D Realization of shielded area 
Selecting deferent cost function constants is a variable that can be depended on the various factors based on the region in which the shielding design is performed, such as monetary factors or availability of specific materials. According to this, a table with different constants and their consequent costs based on (7) is provided (Table I). This table is based on the first scenario in which the height of the wires is the minimum possible height and five wires are employed according to the computer software solution. Increasing the height of the wires leads to a decrease in their number. In this problem, in order to protect all the equipment with just two wires, the final result will be as depicted in Figure 9.

TABLE I. COSTS OF THE FIRST SCENARIO (\$)

\begin{tabular}{|c|c|c|c|c|c|c|}
\hline \multicolumn{2}{|c|}{} & \multicolumn{5}{|c|}{$\boldsymbol{\alpha}$} \\
\cline { 2 - 7 } \multicolumn{2}{|c|}{} & 10 & 20 & 30 & 40 & 50 \\
\hline \multirow{4}{*}{$\beta$} & 10 & 170 & 220 & 270 & 320 & 370 \\
\cline { 2 - 7 } & 20 & 290 & 340 & 390 & 440 & 490 \\
\cline { 2 - 7 } & 30 & 410 & 460 & 510 & 560 & 610 \\
\cline { 2 - 7 } & 40 & 530 & 580 & 630 & 680 & 730 \\
\cline { 2 - 7 } & 50 & 650 & 700 & 750 & 800 & 850 \\
\hline
\end{tabular}

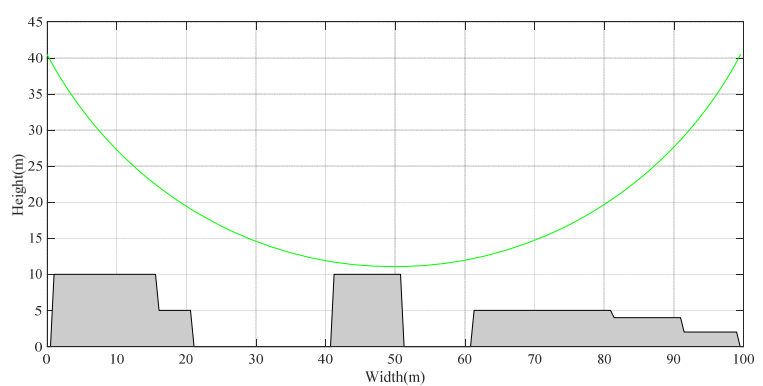

Fig. 9. Protection with two wires

In this scenario, the final cost of designing this protection system is stated in Table II.

TABLE II. COST OF THE SECOND SCENARIO (\$)

\begin{tabular}{|c|c|c|c|c|c|c|}
\hline \multicolumn{2}{|c|}{} & \multicolumn{5}{|c|}{$\boldsymbol{\alpha}$} \\
\cline { 3 - 7 } & 10 & 20 & 30 & 40 & 50 \\
\hline \multirow{4}{*}{$\beta$} & 10 & 420 & 440 & 460 & 480 & 500 \\
\cline { 2 - 7 } & 20 & 820 & 840 & 860 & 880 & 900 \\
\cline { 2 - 7 } & 30 & 1220 & 1240 & 1260 & 1280 & 1300 \\
\cline { 2 - 7 } & 40 & 1620 & 1640 & 1660 & 1680 & 1700 \\
\cline { 2 - 7 } & 50 & 2020 & 2040 & 2060 & 2080 & 2100 \\
\hline
\end{tabular}

The optimal configuration derived is based on $\alpha=40$ and $\beta=10$ which are obtained by an expert in the region in which the substation has been located. The final output of the algorithm is depicted in Figure 10. In this design, three shielding wires have been applied and the height of the wires from the ground is 15 meters. Thus, the total cost of this design is found to be the lowest. As a final step, 3-D realization of Figure 10 is depicted in Figure 11.

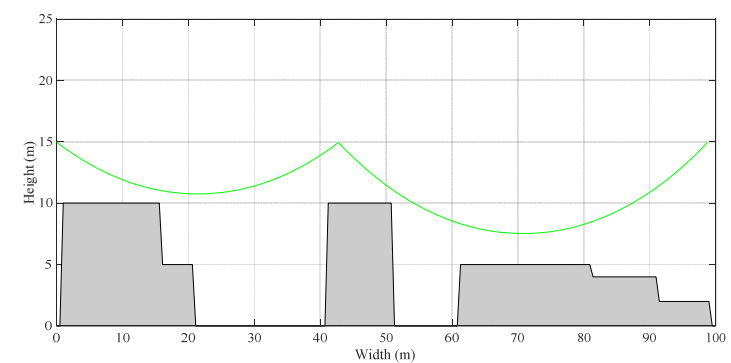

Fig. 10. Optimal shielding protection design

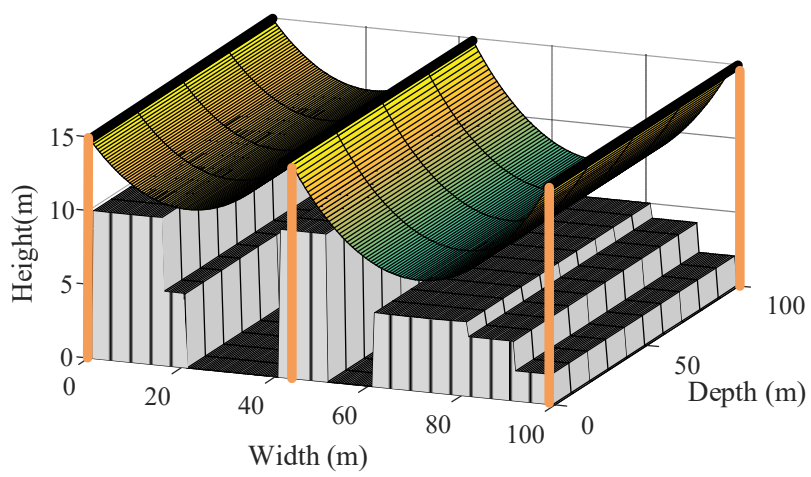

Fig. 11. 3-D Realization of the optimal design

\section{CONCLUSION}

In this paper, an algorithm has been developed in MATLAB in order to design a lightning shielding protection system for substations using shielding wires. Young's equations are employed to determine the value of the different strike distances in the design process. As a significant factor in design, an economic constraint has been defined and this factor determines the number of the shielding wires to be employed and their height from the ground. Finally, the algorithm has been applied to a sample substation and simulation results are shown and discussed.

\section{REFERENCES}

[1] P. Sen, "Understanding direct lightning stroke shielding of substations", Power Syst. Eng. Res. Center, PSERC Seminar, Colorado, November 6, 2001

[2] F. A. M. Rizk, "Modeling of substation shielding against direct lightning strikes", IEEE Trans. Electromagn. Compat., Vol. 52, No. 3, pp. 664 675,2010

[3] K. L. V. Dung, "Lightning protection systems design for substations by using masts and Matlab", International Journal of Mathematical, Computational, Physical, Electrical and Computer Engineering, Vol. 4, No. 5, pp. 562-566, 2010

[4] F. A. M. Rizk, "Exposure of overhead conductors to direct lightning strikes: modeling of positive streamer inhibition", IEEE Trans. Power Deliv., Vol. 26, No. 2, pp. 1156-1165, 2011

[5] S. Dedeoglu, S. Yilmaz, "Design and implementation of a programmable high-voltage impulse measurement system", Turkish Journal of Electrical Engineering \& Computer Sciences, Vol. 22, pp. $262-275,2014$ 
[6] A. M. Mousa, "Shielding of high-voltage and extra-high-voltage substations", IEEE Trans. Power Appar. Syst., Vol. 95, No. 4, pp. 13031310,1976

[7] A. M. Mousa, R. J. Wehling, "A survey of industry practices regarding shielding of substations against direct lightning strokes," IEEE Trans. Power Deliv., Vol. 8, No. 1, pp. 38-47, 1993

[8] A. Hileman, Insulation coordination for power systems, CRC Press, 1999

[9] A. H. A. Bakar, C. K. Tan, A. Z. Abidin, P. J. Khai, H. Mokhlis, H. A. Illias, "Comparative Study on substation shielding due to direct lightning strokes", J. Power Energy Eng., Vol. 2, No. 4, pp. 600-611, 2014

[10] A. Mousa, "A computer program for designing the lightning shielding systems of substations," IEEE Transactions on Power Delivery, Vol. 6, No. 1, pp. 143-152, 1991

[11] J. McDonald, Electric Power Substations Engineering, CRC Press, 2012

[12] IEEE Std 998-1996, IEEE Guide for Direct Lightening Stroke Shielding of substations, 1996 